\title{
ARTICLE \\ Donepezil attenuates vascular dementia in rats through increasing BDNF induced by reducing HDAC6 nuclear translocation
}

\author{
Wen-xuan Jian ${ }^{1,2}$, Zhao Zhang ${ }^{3}$, Jia-hong Zhan ${ }^{1,2}$, Shi-feng $\mathrm{Chu}^{3}$, Ye Peng ${ }^{4}$, Ming Zhao ${ }^{5}$, Qi Wang ${ }^{1,2}$ and Nai-hong Chen ${ }^{1,3,4}$
}

\begin{abstract}
Vascular dementia (VD) is the second most common dementia disease after Alzheimer's diseases (AD) in the world. Donepezil is used to treat mild to moderate $A D$, and it has been shown to treat cognitive impairment and memory deficits caused by VD. However, the action mechanism of donepezil against VD has not been clarified. In this study, a bilateral common carotid artery occlusion (BCCAO) model was established in rats to simulate the pathology of VD. Two weeks after the surgery, the rats were administered donepezil $\left(10 \mathrm{mg} \cdot \mathrm{kg}^{-1} \cdot \mathrm{d}^{-1}, \mathrm{ig}\right)$ for 3 weeks, and then subjected to behavioral tests. We showed that donepezil treatment significantly improved the performance of BCCAO rats in Morris Water Mazes test and Step-down test. Furthermore, we showed that donepezil treatment significantly attenuated neurodegeneration and restored the synapse dendritic spines density in cortex and hippocampus. We revealed that donepezil treatment significantly increased BDNF expression in cortex and hippocampus. Interestingly, donepezil treatment significantly decreased nuclear translocation of HDAC6 and the binding between HDAC6 and BDNF promoter IV in cortex, but not in the hippocampus. The attenuated neurodegeneration by donepezil in cortex and hippocampus might due to the reduced ROS levels and increased phosphorylation of AMPK, whereas increased phosphorylation of AKT was only detected in cortex. In conclusion, our results demonstrate that donepezil attenuates neurodegeneration in cortex and hippocampus via increasing BDNF expression; the regulation of donepezil on HDAC6 occurred in cortex, but not in the hippocampus. This study further clarifies the pharmacological mechanism of donepezil, while also emphasizes the promising epigenetic regulation of HDAC6.
\end{abstract}

Keywords: donepezil; vascular dementia; bilateral common carotid artery occlusion (BCCAO) model; neurodegeneration; cortex; hippocampus; BDNF; HDAC6; epigenetic regulation

Acta Pharmacologica Sinica (2020) 41:588-598; https://doi.org/10.1038/s41401-019-0334-5

\section{INTRODUCTION}

Vascular dementia (VD) is the second most common dementiarelated disease in the world after Alzheimer's disease (AD) [1, 2]. The risks of VD increase with age, and the severity of VD depends on injury to vessels and cerebral regions. Deficits in information processing, trouble speaking or understanding, vision loss, cognitive impairment, and memory loss are symptoms of VD [3]. There are no drugs specific for VD according to the FDA; however, drugs used for AD have a certain efficacy on VD in the clinic [4]. Donepezil is an acetylcholinesterase inhibitor that is approved by FDA for mild to moderate AD. However, the mechanism by which donepezil attenuates VD is not fully understood. Therefore, the further study of the mechanism of the protective effect of donepezil against VD would be meaningful for understanding its ability to treat VD and would provide theoretical reference for the development and research of new drugs for VD.

Brain-derived neurotrophic factor (BDNF) is widely distributed in the central nervous system (CNS) and it is an important neurotrophic factor in the cerebrum. BDNF promotes synaptic activity, increases synaptic plasticity, and protects against neurodegeneration $[3,5,6]$. The transcription of BDNF is regulated by several promoters, of which the BDNF promoter IV is a key factor for neuronal activity [6]. BDNF is obviously reduced in the cerebrum of $A D$ and VD patients [7]. A clinical trial indicated that VD patients treated with fluoxetine for 12 weeks exhibited better MMSE scores than those of the placebo-treated patients, who exhibited an increase in BDNF in the serum [8]. Therefore, BDNF is considered a key target for VD therapy. In an in vivo model of $A D$, donepezil mediated BDNF via BDNF/TRKB signaling [9]. Donepezil also inhibited miR-206-3p and increased the expression of BDNF [10], implying that donepezil improved $A D$ in an epigenetic manner. However, few studies have explained the manner in which donepezil regulates BDNF in VD.

Histone deacetylases (HDACs) are a class of enzymes that alter DNA. Recent studies have found changes in HDACs in neurodegenerative diseases [11]. The class II HDAC HDAC6 is related to

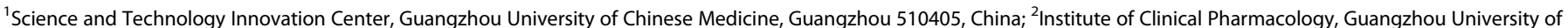

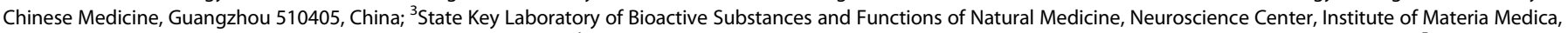

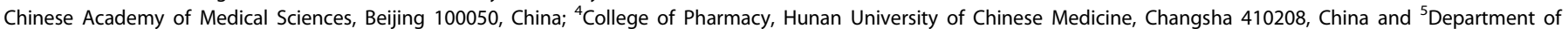
Pharmacy, Beijing Hospital, National Center of Gerontology, Beijing 100730, China

Correspondence: Nai-hong Chen (chennh@imm.ac.cn)

Received: 12 July 2019 Accepted: 18 November 2019

Published online: 8 January 2020 
neurodevelopmental and neurodegenerative diseases and is upregulated in the cortex and hippocampus in AD patients [12]. In an in vivo study using an HDAC6 inhibitor, neuronal damage caused by A-beta was attenuated [13]. However, studies on HDAC6 in VD are still insufficient. In addition, it is possible to explain the mechanism of the effect of donepezil on VD by epigenetics.

According to the recent studies, BDNF is regulated by HDAC6 nuclear translocation. In AD, HDAC6 is translocated into the nucleus and binds to the BDNF promoter, thus reducing the expression of BDNF [14]. Our study aimed to determine whether donepezil is able to regulate BDNF via HDAC6 nuclear translocation in VD to further clarify the pharmacological mechanism of donepezil, to provide a deeper understanding of the donepezilmediated increase in the expression of BDNF, and to provide a theoretical foundation for the development of drugs for VD.

\section{MATERIALS AND METHODS}

Animals

Male Sprague-Dawley rats weighing $250 \pm 10 \mathrm{~g}$ were supplied by SPF Laboratories (Beijing, China) and were housed in groups of four per cage at a temperature of $23 \pm 1{ }^{\circ} \mathrm{C}$ on a 12-h light-dark cycle. Food and water were provided ad libitum. All experiments were performed in accordance with the guidelines established by the National Institutes of Health Guide for the Care and Use of Laboratory Animals (NIH Publications No. 8023, revised 1978) and were approved by the Animal Care Committee of the Peking Union Medical College and Chinese Academy of Medical Sciences (Beijing, China).

\section{Surgical procedure}

Bilateral common carotid artery occlusion (BCCAO) was established to study the cognitive dysfunction and neurodegeneration caused by chronic cerebral hypoperfusion $(\mathrm{CCH})$. The BCCAO surgery was performed as previously conducted in our laboratory [1]. Thirty-two Sprague-Dawley rats were randomly divided into 2 groups, namely, the sham group $(n=8)$ and the BCCAO group $(n=24)$. All rats were allowed to drink freely but were fasted for $12 \mathrm{~h}$ before surgery. Before surgery, each rat was anesthetized with a mixture of $5 \%$ isoflurane and $95 \%$ oxygen in a covered tank. During the operation, the rats were given continued anesthesia with a mixture of $3 \%$ isoflurane and $97 \%$ oxygen through a respiratory mask. In the BCCAO group, a 1.5- to $2-\mathrm{cm}$ incision was made in the epidermis along the cervical midline, and the muscles were bluntly separated until the common carotid artery was exposed. Two sections of the common carotid artery were ligated with No. 0 silk thread, and the intermediate vessel was occluded with an electric coagulator. The other side of common carotid artery was treated similarly. After the wound was sutured, the rats were given free access to water and food. The sham group underwent the same operation as the BCCAO group, but the common carotid artery was not ligated or blocked. Fifteen days after surgery, the BCCAO group was randomly divided into the BCCAO model group $(n=12)$ and the donepezil-treated group $(n=12)$.

Drug administration and experimental design

Donepezil was purchased from Eisai (H20070181, Eisai, Suzhou, China) and dissolved in $0.5 \%$ CMC-Na at a concentration of $1 \mathrm{mg} / \mathrm{mL}$. The drug was administered orally once a day starting 2 weeks after the surgery (d 15). The donepezil-treated group received $1 \mathrm{~mL} / 100 \mathrm{~g}$ donepezil-0.5\% CMC-Na. The sham group and BCCAO group received $1 \mathrm{~mL} / 100 \mathrm{~g} \mathrm{0.5 \%} \mathrm{CMC-Na.} \mathrm{Behavioral}$ tests, including 7 days of the Morris water maze test (days 35-41) and 2 days of the step-down test (days 43-44), were performed 3 weeks after drug administration (day 35 ). The timeline of the experiment is shown in Fig. 1 a.
Morris water maze test

The Morris water maze test was performed as previously described. The water maze device was a black circular tank with a diameter of $120 \mathrm{~cm}$, a height of $50 \mathrm{~cm}$, and a water depth of $\sim 30 \mathrm{~cm}$. A black platform with a diameter of $10 \mathrm{~cm}$ was placed in the water maze with the top of the platform $\sim 2 \mathrm{~cm}$ below the water. The tank was divided into 4 equal parts (areas A-D) and the platform area (area T) by a recording system (SMART Panlab, Harvard Bioscience, Shanghai, China). The platform was located in area $\mathrm{A}$. In phase I, the training phase (day 35 ), each rat was gently placed into the water maze in area $C$ facing the tank wall for $90 \mathrm{~s}$. If the platform was not found during the training, the rat was guided to the platform and kept there for $20 \mathrm{~s}$ for observation and learning. During phase II, the latency to platform phase (days 36-40), each rat was gently placed into the water maze in area C facing the tank wall and searched for the platform. The system recorded the route of the rat in the maze. If the rat successfully found the platform and stayed in area $T$ for $2 \mathrm{~s}$, the system recorded the time. If the platform was not found within $90 \mathrm{~s}$, the time was recorded as $90 \mathrm{~s}$, and the rat was guided to the platform and kept there for $20 \mathrm{~s}$ for observation and learning. In phase III, the platform-crossing phase (day 41), the platform was removed, and each rat was gently placed into the water maze in area $C$. The number of times the rat crossed area $T$ in $90 \mathrm{~s}$ was recorded. The learning ability and spatial memory ability of the rats were evaluated based on the latency to find the platform and the number of platform crossings.

\section{Step-down test}

The step-down test was performed with the YLS-17B apparatus (Sansbio, Nanjing, China). In stage I, an insulated platform was placed in the middle of the dark box, and each rat was placed on the grid at the bottom of the dark box, which was electrified for $2 \mathrm{~min}$ at $50 \mathrm{~V}$. If the SD rat failed to jump on the platform within $30 \mathrm{~s}$, it was guided to the platform and kept there for $2 \mathrm{~min}$. In stage II, each rat was placed on the insulated platform, and the grid was electrified for $2 \mathrm{~min}$. The system automatically recorded the number of shocks that the rats received. One electric shock recorded as one error, and the working memory of the SD rats was evaluated based on the number of errors.

\section{Nissl staining and DHE staining}

Rats were randomly selected, anaesthetized with chloral hydrate (350 mg/kg, ip), and perfused with PBS and then 4\% paraformaldehyde. The whole brain was removed after perfusion, fixed, and preserved with $4 \%$ paraformaldehyde. The fixed brain tissue was sent to Servicebio (Wuhan, China) for paraffin sectioning.

Nissl staining was completed by Servicebio. The tissue sections were visualized with a light microscope and captured with Image Pro Plus. The number of normal cells and Nissl-stained cells was calculated using ImageJ Cell Counter. The percentage of neurodegeneration was used to evaluate nerve damage. The following formula was used: neurodegeneration percentage $=$ pathological neuronal cells/total cells in the cortex or hippocampus in the field.

For DHE staining, after dewaxing and rehydration, tissue sections were incubated for antigen retrieval for $10 \mathrm{~min}$ and then incubated with dihydroethidium $(100 \mu \mathrm{M}$, Molecular Probes, Sigma, D7008) at room temperature for four hours in the dark. The slides were rinsed three times with PBST, mounted with glycerin, and coverslipped. The tissue sections were visualized with a fluorescence microscope and captured with Image Pro Plus. The IOD was measured by Image Pro Plus.

\section{Golgi-Cox staining}

Rats were randomly selected for Golgi-Cox staining. Golgi-Cox staining was carried out with a HITO Golgi-Cox OptimStain ${ }^{T M}$ Prekit, and the experiment was performed as described below. 


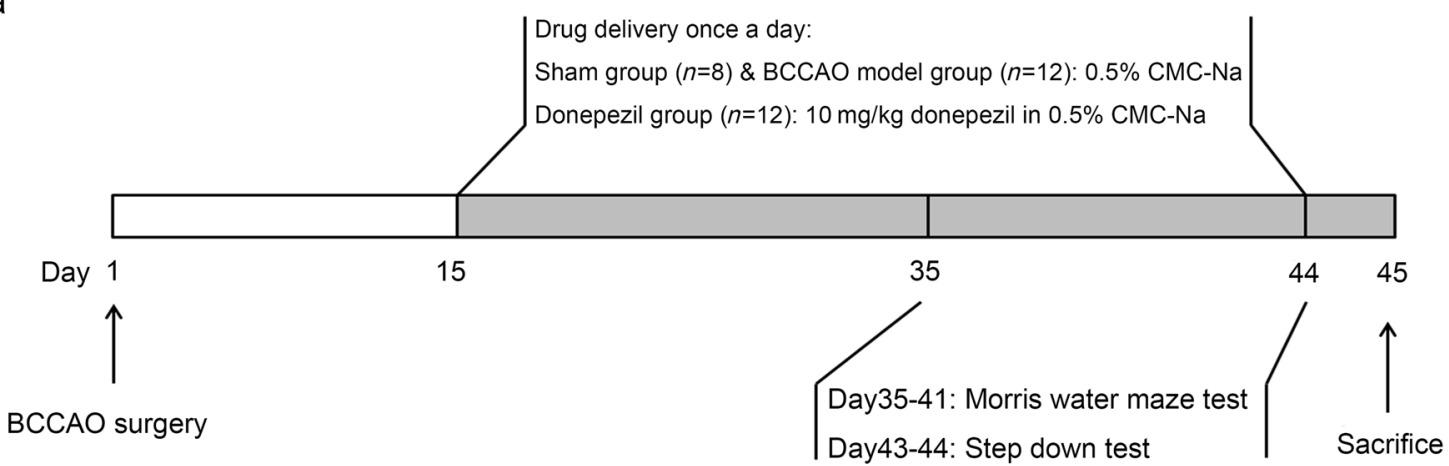

b

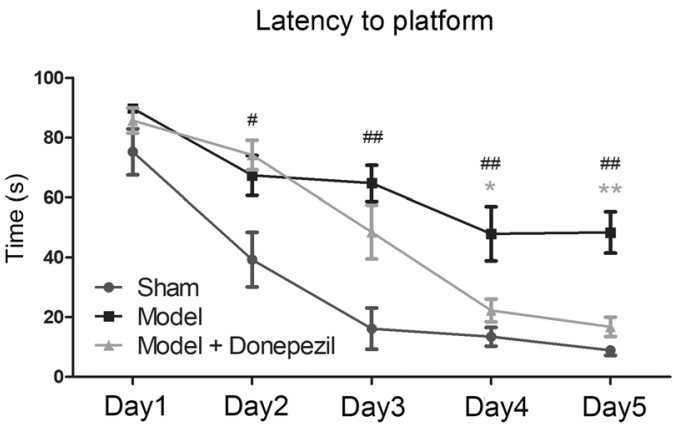

d

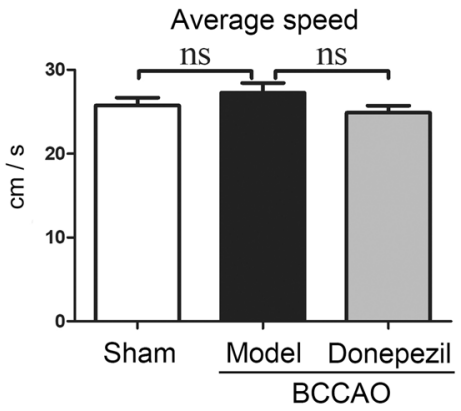

C

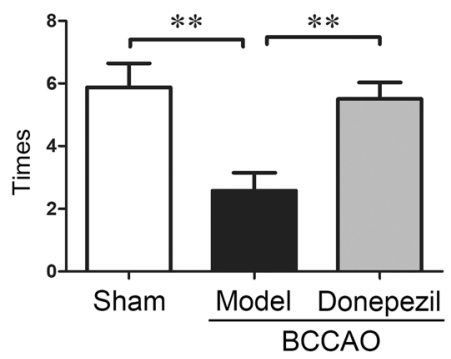

e

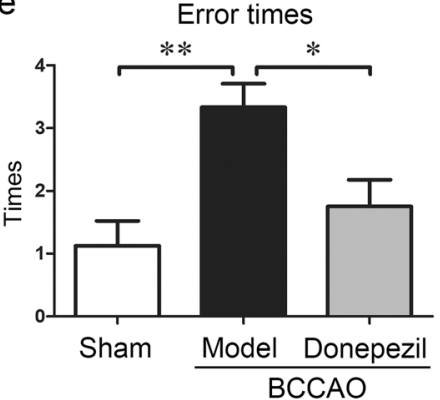

Fig. 1 Donepezil improved performance in the MWM test and step-down test in BCCAO rats. a The day of BCCAO surgery was regarded as the first day of the experiment. Drugs were administered once a day beginning on $\mathrm{d} 15$. Drug delivery and behavioral tests were performed in the sham group $(n=8)$, BCCAO model group $(n=12)$, and donepezil-treated group $(n=12)$. The MWM test was carried out from days 35 to 41 , and the step-down test was carried out from days 43 to 44 . All rats were killed on day 45 . The significance of the differences in platform escape latency was analyzed by two-way ANOVA. The data are shown as the mean \pm SEM. ${ }^{\#} P<0.05$ and ${ }^{\# \#} P<0.01$, the model group vs the sham group. ${ }^{*} P<0.05$ and ${ }^{* *} P<0.01$, the model + donepezil-treated group vs the model group. $\mathbf{b}$ The platform escape latency represented the time for each rat to find the platform in phase II of the MWM test. c The number of platform crossings represented the number of times each rat entered the platform zone in phase III of the MWM test. $\mathbf{d}$ The average speed represented the swimming speed of the rats in phase III of the MWM test. e The number of errors was automatically evaluated by the system. An error was counted when a rat on the insulation platform touched the grid and received an electric shock, and the total number of times that the rats received an electric shock in 2 min represented the number of errors. The significance of differences in platform escape latency was analyzed by two-way ANOVA, and the significance of the rest of the data was analyzed by one-way ANOVA. The data are shown as the mean \pm SEM. ${ }^{*} P<0.05$ and ${ }^{* *} P<0.01$.

The rats were killed, and the whole brains were removed. After washing with $\mathrm{ddH}_{2} \mathrm{O}$, the whole brains were incubated in solution 1 and 2 for two weeks and then solution 3 for 1 week. Tissue sections (100- $\mu$ m-thick) were prepared with a cryostat microtome (Leica CM3050s). The tissue sections were stained with solution 4 and 5 , and the slices were mounted with neutral balsam and coverslipped after dehydration. The tissue sections were visualized with a light microscope and captured with Image Pro Plus. ImageJ was used for nerve analysis. When selecting cortical neural cells, we selected the same area as that used for Nissl staining for dendritic spine photography. In the hippocampus, hippocampal neural pyramidal cells were selected for imaging. Dendrites without intersections and with a clean background were selected for dendritic spine photography and statistics.
Immunofluorescence

After dewaxing and rehydration, tissue sections were incubated for antigen retrieval for $10 \mathrm{~min}$ and with Triton X-100 for $10 \mathrm{~min}$. The sections were blocked with $5 \%$ BSA, incubated in a humidified chamber for $1 \mathrm{~h}$ at room temperature, and incubated with antibodies against target proteins in a humidified chamber overnight at $4{ }^{\circ} \mathrm{C}$. The slides were rinsed three times with PBST and incubated with immunofluorescence secondary antibodies and Hoechst in the dark at room temperature for $2 \mathrm{~h}$. The slides were rinsed three times with PBST, mounted with glycerin, and coverslipped. The tissue sections were visualized and captured with a confocal microscope.

The following antibodies were used: HDAC6 (A11259, Abclonal), Alexa Fluor ${ }^{\mathrm{TM}}$ 488-conjugated donkey anti-rabbit lgG $(\mathrm{H}+\mathrm{L})$ (A21206, Invitrogen), and Hoechst 33342 solution (H342, Dojindo). 
Western blotting

Rats were killed, and the whole brains were removed. The cortex and hippocampus were collected, and total protein was extracted with RIPA lysis buffer. Nuclear proteins and cytosolic proteins were extracted with a Nuclear-Cytosol Extraction Kit (P1200, Applygen Technologies Inc, Beijing, China). The concentration of total protein was measured by BCA assay. SDS-PAGE gels $(10 \%$ or $12 \%)$ was used for gel electrophoresis according to the molecular weight of the target protein, and the proteins were transferred to $0.45-\mu \mathrm{m}$ PVDF membrane by wet electroblotting. The protein bands were visualized and captured using an ImageQuant LAS $4000 \mathrm{mini}$, and the integrated densities were analyzed by ImageJ.

The following antibodies were used: p-AMPK (sc-101630, Santa Cruz), AMPK (sc-19138, Santa Cruz), p-AKT (sc-33437, Santa Cruz), AKT (sc-8312, Santa Cruz), BDNF (sc-546, Santa Cruz), HDAC6 (A11259, Abclonal), $\beta$-actin (AC037, Abclonal), and PCNA (sc-7907, Santa Cruz).

Total mRNA extraction and qRT-PCR

Total mRNA was extracted with TRIzol, and the concentration of total mRNA was measured with a NanoDrop 2000 spectrophotometer. mRNA was reverse transcribed into cDNA with a reverse transcription kit (TransScript II One-Step gDNA Removal and cDNA Synthesis SuperMix, TransGen Biotech, Beijing, China). The expression of the target gene was measured by qPCR, and GAPDH as used as a reference. The primer sequences were as following: BDNF (forward: 5'-GAAGCTCAACCGAAGAGCTAAA-3', reverse: $5^{\prime}$-AGCCTTCATGCAACCGAAGTA-3') and GAPDH (forward: 5'-CTGGAAGATGGTGATGGGTT-3', reverse: 5'-ATGACTCTACCCAC GGCAAG-3').

\section{Chromatin Immunoprecipitation and PCR}

Chromatin immunoprecipitation was performed with a CHIP assay kit (P2078, Beyotime, Shanghai, China). The experiment was performed as described below. The tissues were fully ground in liquid nitrogen, $1 \%$ formaldehyde PBS solution was added, the tissues were incubated at $37^{\circ} \mathrm{C}$ for $10 \mathrm{~min}$, and the binding of proteins and the genome was determined. Glycine solution was added, the tissues were incubated at room temperature for $5 \mathrm{~min}$ and centrifuged, and the tissue samples were collected. The samples were centrifuged and washed with PMSF-PBS. The samples were dissolved in lysis buffer containing PMSF and SDS for $10 \mathrm{~min}$ on ice, and then the samples were sonicated. The samples were centrifuged and dissolved in ChIP Dilution Buffer. The input was removed, Protein $A+G$ Agarose/Salmon Sperm DNA was added to the remainder, and the samples were mixed at $4{ }^{\circ} \mathrm{C}$ for $30 \mathrm{~min}$. The samples were centrifuged, the supernatant was collected, primary antibody was added, and the samples were mixed at $4{ }^{\circ} \mathrm{C}$ overnight. Protein $\mathrm{A}+\mathrm{G}$ Agarose/Salmon Sperm DNA was added, and the samples were mixed at $4{ }^{\circ} \mathrm{C}$ for $60 \mathrm{~min}$ to allow binding to the primary antibody complex. The samples were centrifuged, the precipitate was washed and dissolved with $T E$, and the genome bound to the primary antibody was obtained. A DNA purification kit was used to purify the genomic samples for PCR or qPCR detection.

Takara LA-Taq (RR02MA, TaKaRa Bio, Osaka, Japan) was used for PCR amplification. The primers, which were used in a previous study [15], were as follows: rat BDNF (rBDNF) promoter I (forward: 5'-ACGTCCGCTGGAGACCCTTAGT-3', reverse: 5'-GGCAGCCTCTCT GAGCCAGTTA-3'), rBDNF promoter III (forward: 5'-TAGGTGA GAACCTGGGGCAA-3', reverse: 5'-CTTGAGCTTCCCCAACCTCG-3'), rBDNF promoter IV (forward: 5'-ATGCAATGCCCTGGAACGGAA-3', reverse: 5'-CGGTGAATGGGAAAGTGGGTGG-3'), and rBDNF promoter VI (forward: 5'-CGCTGTCTGACCAATCGAAG-3', reverse: $5^{\prime}$-GTTTCCTTCTCCAAGCCGGG-3'). The PCR amplification products were detected on a $1 \%$ agarose gel.
Statistical analysis

Each set of statistical data had three or more samples. All data are expressed as the mean \pm SEM. Statistical analyses were performed using GraphPad Prism 5. Group comparisons were performed by one-way ANOVA with Bonferroni's multiple comparison test or two-way ANOVA with Bonferroni's multiple comparison test. $P<$ 0.05 was considered significant.

\section{RESULTS}

Donepezil attenuated the cognitive impairment and memory dysfunction of BCCAO rats

To investigate cognitive recovery induced by donepezil in $\mathrm{CCH}$, we evaluated learning and memory functions by analyzing escape latency and the number of platform crossings in the MWM test. All rats showed a progressive reduction in escape latency (Fig. 1b). The escape latency of the sham group rats was statistically shorter than that of the BCCAO model group rats from days $2-5$ in phase II of the MWM test $(P<0.05$ from day 2$)$. The escape latency of the donepezil group was shorter than that of the BCCAO model group from $\mathrm{d} 4(P<0.05$ at day $4, P<0.01$ at day 5$)$. We examined the frequency of platform zone crossings in phase III of the MWM test (Fig. 1c) and found that the BCCAO model group crossed the platform zone less frequently than the sham group $(P<0.01)$. Donepezil treatment increased the frequency compared with that of the BCCAO model group $(P<0.01)$. There was no difference in swimming speed (Fig. 1d) between all groups $(P>0.05)$, which indicated that BCCAO did not impair motor ability. We next examined working memory by the step-down test. The number of electric shocks received by the rats was used as a measure of the number of errors. In the step-down test (Fig. 1e), the BCCAO model group showed a greater error frequency than the sham group $(P<0.01)$, and donepezil-treated rats exhibited a reduced error frequency compared with that of the BCCAO model group $(P<0.05)$. Taken together, the results of the MWM test and stepdown test validated the establishment of cognitive impairment and memory degeneration in the BCCAO model. Furthermore, these results indicated that donepezil mitigated learning and memory impairment caused by BCCAO.

Donepezil attenuated neurodegeneration caused by BCCAO and increased BDNF expression in the cerebrum

Nissl staining was carried out to investigate neurodegeneration in the cerebrum. Representative microphotographs of Nissl staining are shown in Fig. 2a, c. The cortex was captured with a light microscope with a $\times 100$ lens (Fig. 2a), and the hippocampus was captured with a light microscope with a $\times 200$ lens (Fig. 2c). Neuronal pathological changes, including disarrangement of neurons, nuclear shrinkage, and dark staining of neuronal cells, were observed in the cortex and hippocampus in the BCCAO model group. Upon treatment with donepezil, neurodegeneration was attenuated. We counted the total number of neuronal cells and the number of pathological neuronal cells within the visual field and analyzed the percentage of neurodegeneration. As showed in Fig. 2b, d, BCCAO increased neurodegeneration percentage in the cortex and hippocampus compared with that in the sham group (both $P<0.01$ ), while donepezil reduced the neurodegeneration percentage compared with that in the BCCAO group (both $P<0.01$ ).

We next performed Golgi-Cox staining to examine dendritic spine synapse density. Representative microphotographs of dendritic spines in the cortex (Fig. 2e) and hippocampus (Fig. 2f) were captured with a light microscope with a $\times 1000$ oil lens. The number of dendritic spines and lengths of the synapses were recorded, and the dendritic spine density was analyzed. The dendritic length and number of spines were determined by ImageJ. The results showed a reduction in the number of dendritic 

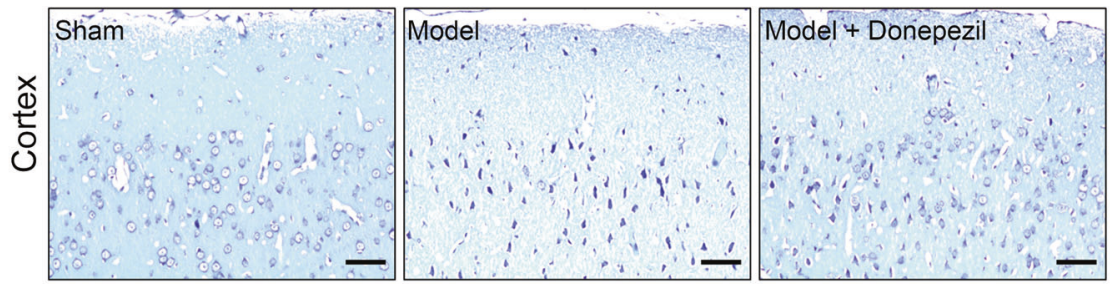

C

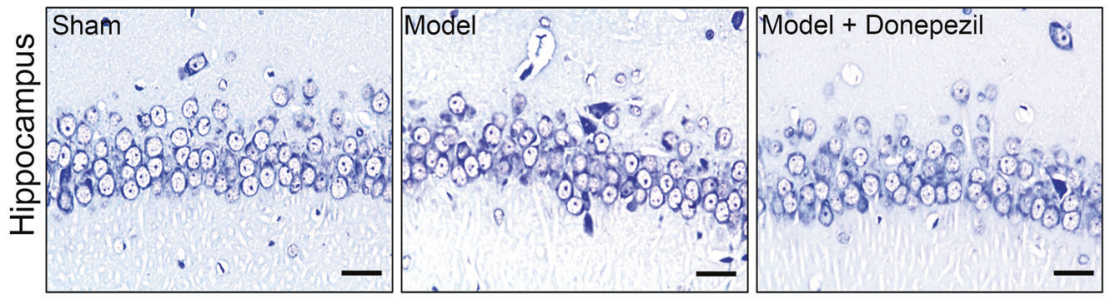

$f$

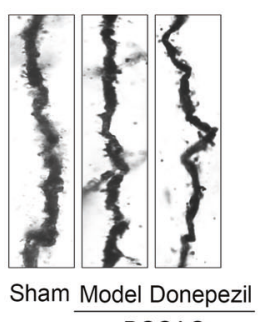

BCCAO

Cortex dendritic density

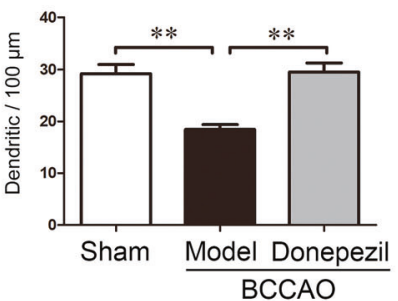

h Hippocampus BDNF mRNA
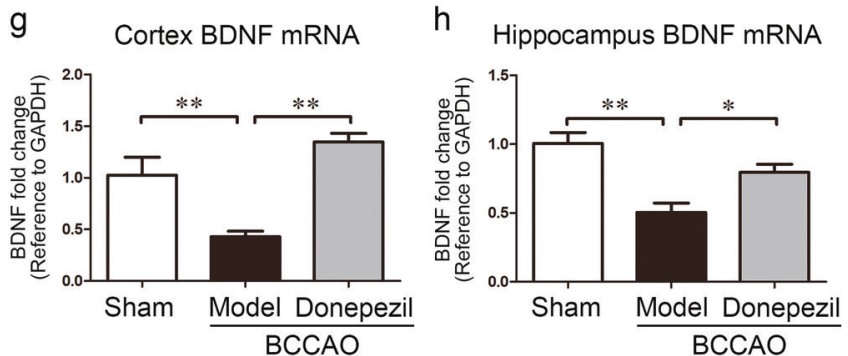

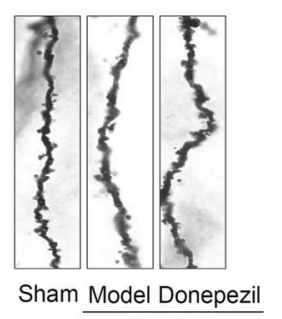

b

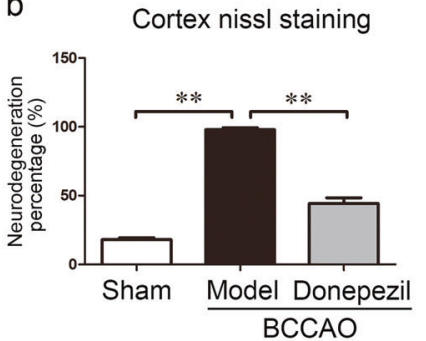

d

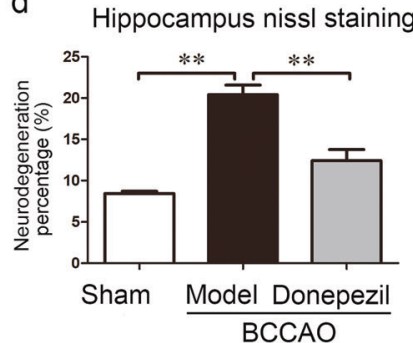

Hippocampus BDNF pyramidal dendritic density

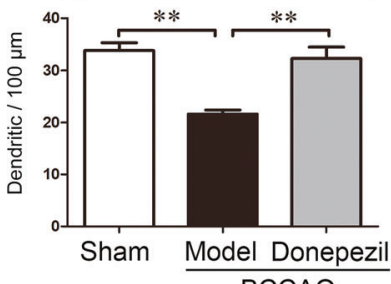

i BCCAO - + +

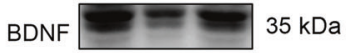

$\beta$-actin $42 \mathrm{kDa}$

Cortex BDNF
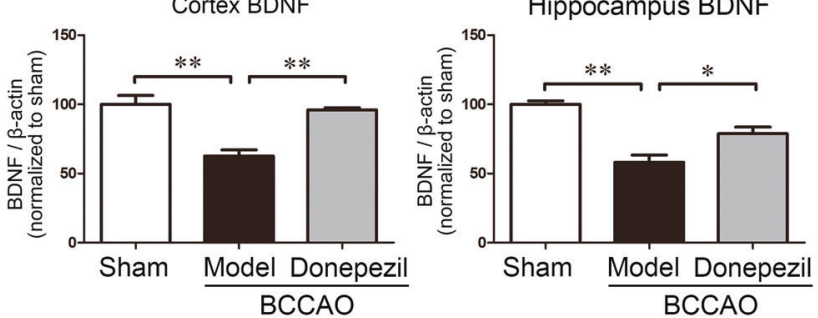

Fig. 2 Donepezil attenuated neurodegeneration and increased the expression of BDNF. a Representative images of Nissl staining in the cortex; scale bar $=100 \mu \mathrm{m}$. b The statistics of the percentage of neurodegeneration in the cortex. c Representative images of Nissl staining in the hippocampus; scale bar $=50 \mu \mathrm{m}$. $\mathbf{d}$ The statistics of the percentage of neurodegeneration in the hippocampus. e, $\mathbf{f}$ Representative images of Golgi-Cox staining of cortical dendrites (e) and hippocampal pyramidal dendrites (f). The histograms beside the images show the statistics of dendritic spine density. $\mathbf{g}, \mathbf{h}$ BDNF mRNA expression in the cortex (g) and hippocampus (h). GAPDH was used as a reference gene, and the data were normalized to the mean of the sham group. $\mathbf{i}$, $\mathbf{j}$ The Western blotting results of BDNF expression in the cortex (i) and hippocampus (j). The histogram below the bands shows the statistics of the integrated density. $\beta$-Actin was used as a reference protein, and the protein expression data were normalized to the mean of the sham group. Significance was analyzed by one-way ANOVA. The data are shown as the mean \pm SEM. ${ }^{*} P<0.05$ and ${ }^{* *} P<0.01$.

spines density in the cortex and hippocampus in the BCCAO model group compared with that in the sham group (both $P<$ $0.01)$. Treatment with donepezil enhanced dendritic spine density compared with that in the BCCAO group (both $P<0.01$ ). Lowmagnification microscopic images are presented in the Supplemental Fig. 1a, b.

BDNF plays an important role in neuronal formation, protects neuronal cells and maintains neuroplasticity [16]. We examined the mRNA expression and protein expression of BDNF in the cortex and hippocampus by qPCR and Western blotting. As reflected in Fig. 2g, h, BCCAO reduced mRNA expression in the cortex and hippocampus compared with that in the sham group (both $P<0.01$ ), while donepezil restored the expression of BDNF mRNA ( $P<0.01$ in cortex, $P<0.05$ in hippocampus). As reflected in Fig. $2 \mathrm{i}$, j, compared with the sham group, the BCCAO model group had a pronounced reduction in the expression 

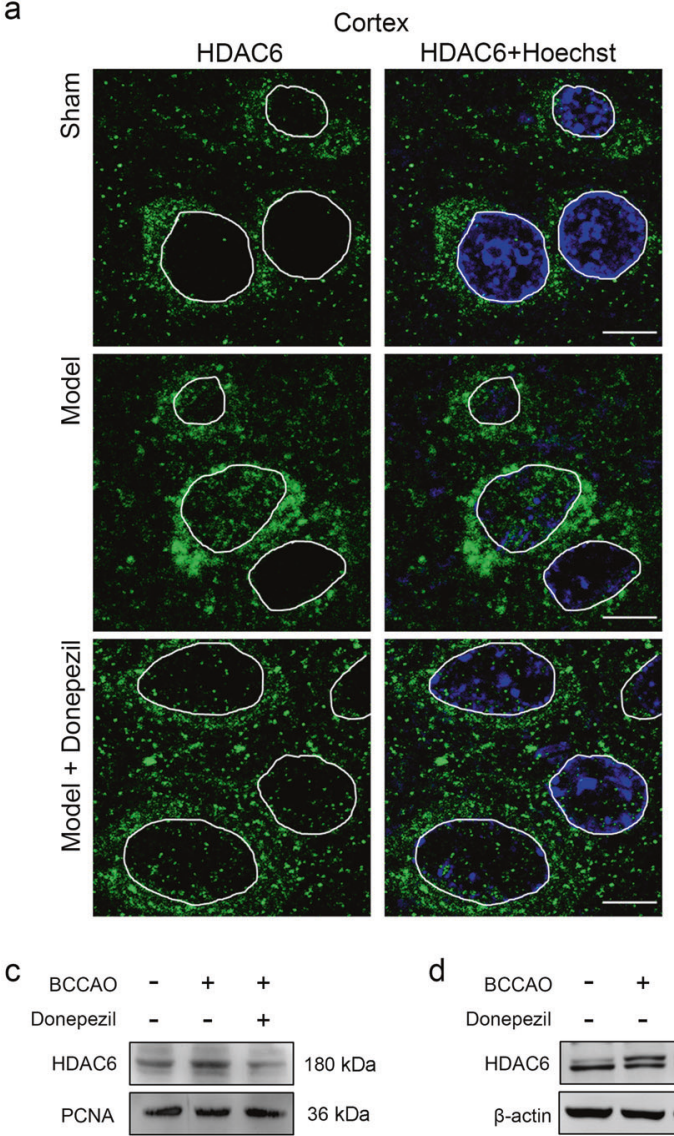

Cortex nuclear HDAC6
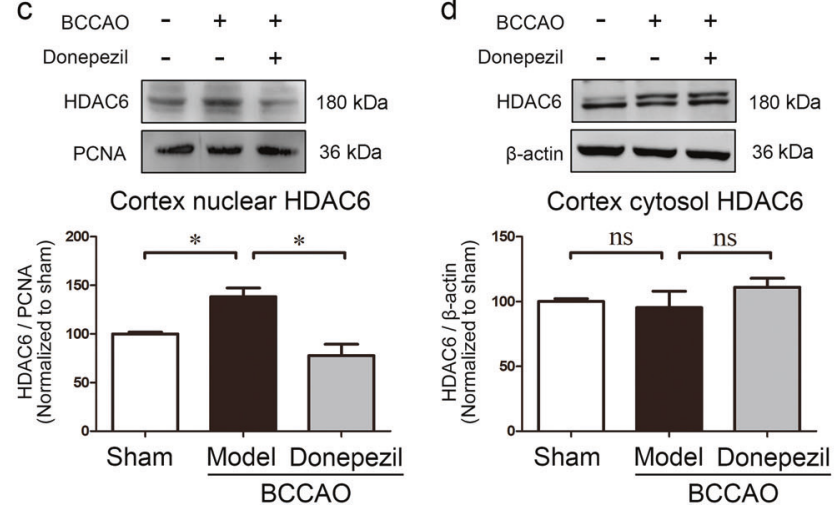

Cortex cytosol HDAC6

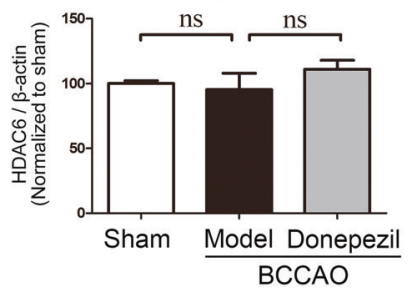

b

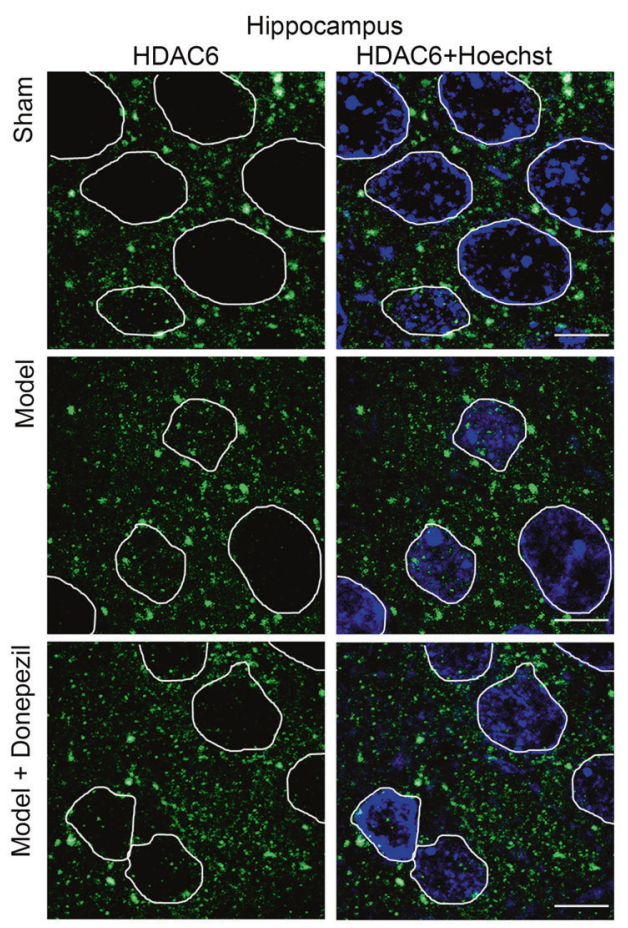

e

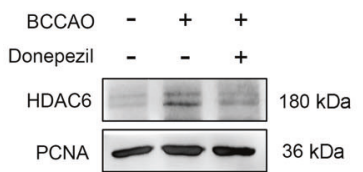

Hippocampus nuclear HDAC6

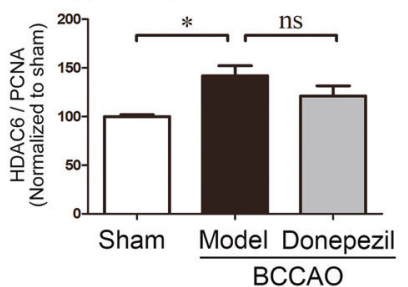

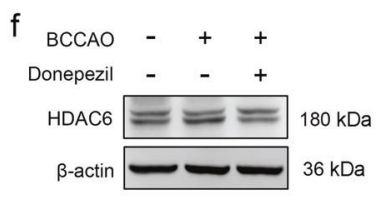

Hippocampus cytosol HDAC6

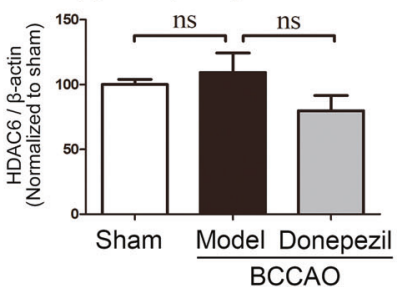

Fig. 3 Donepezil decreased the nuclear translocation of HDAC6 in the cortex. $\mathbf{a}, \mathbf{b}$ Immunofluorescence was carried out to investigate the translocation of HDAC6. HDAC6 was labeled with green fluorescence, and nuclei were labeled with blue fluorescence by Hoechst. The white line shows the margin of the nucleus; scale bar $=5 \mu \mathrm{m}$. c-f The Western blotting results of HDAC6. The histogram below the bands shows the statistics of the integrated density. PNCA was used as a nuclear reference protein (c, e), and $\beta$-actin was used as a cytosolic reference protein (d, f). Protein expression was normalized to the mean of the sham group. Significance was analyzed by one-way ANOVA. The data are shown as the mean \pm SEM. ${ }^{*} P<0.05$.

of BDNF in both the cortex and hippocampus (both $P<0.01$ ). In addition, donepezil treatment resulted in an enhancement of BDNF expression $(P<0.01$ in the cortex, $P<0.05$ in the hippocampus).

Donepezil reduced the nuclear translocation of HDAC6 in the cortex

Although donepezil restored BDNF mRNA and protein expression in the cortex and hippocampus, the previous results showed that BDNF recovery in the cortex was greater than that in the hippocampus, which implies difference in BDNF epigenetics. The nuclear translocation of HDAC6 is a key step in mediating the expression of BDNF in late onset AD [14]. To observe the distribution of HDAC6 in the cortex and hippocampus, we carried out immunofluorescence for HDAC6 and Hoechst. Representative fluorescence microphotographs of the cortex (Fig. 3a) and hippocampus (Fig. 3b) showed that BCCAO increased the nuclear location of HDAC6, while donepezil reduced the level of HDAC6 in the nucleus. To investigate the separation of HDAC6, we next examined HDAC6 protein expression in the nucleus and cytoplasm. Low-magnification microscopic images were presented in the Supplemental Fig. 1a, b. In the cortex, as shown in Fig. 3c, BCCAO enhanced the level of HDAC6 in the nucleus compared with that in the sham group $(P<0.05)$, and donepezil decreased the level of HDAC6 in the nucleus compared with that in BCCAO model group $(P<0.05)$. In the hippocampus, as shown in Fig. 3e, BCCAO increased the level of HDAC6 in the nucleus compared with that in the sham group $(P<0.05)$, and donepezil decreased the level of HDAC6 in the nucleus compared with that in the BCCAO model group; however, there was no significant difference between the model group and donepezil group $(P>$ $0.05)$. No significant difference in the cytoplasmic protein expression of HDAC6 in the cortex (Fig. 3d) or hippocampus (Fig. 3f) was detected (both $P>0.05$ ). 
a

Cortex HDAC6-ChIP

M Input Sham Model Donepezil IgG

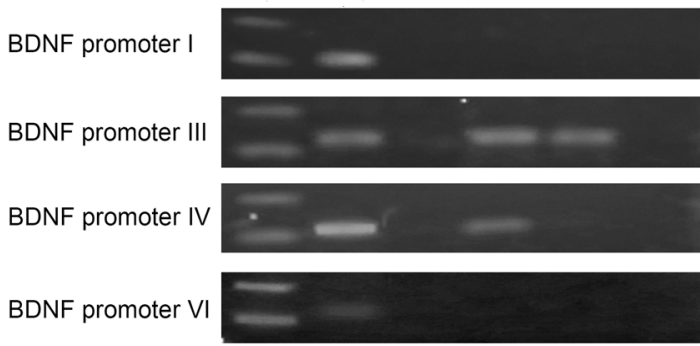

C

Cortex BDNF promoter III

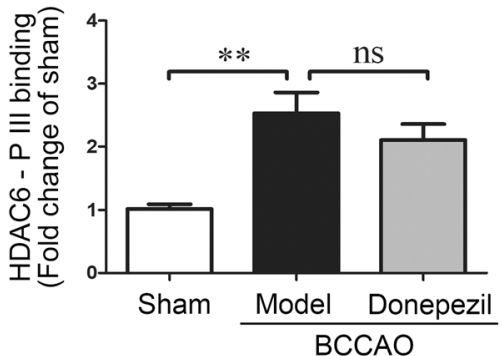

e

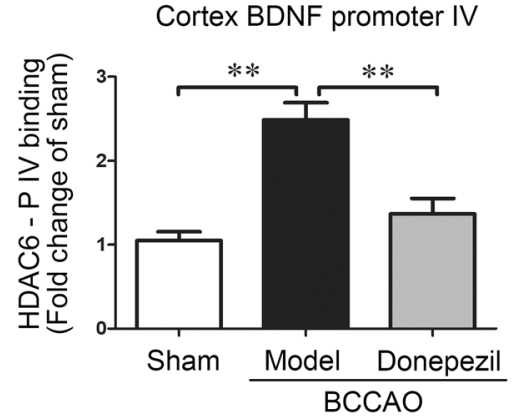

b

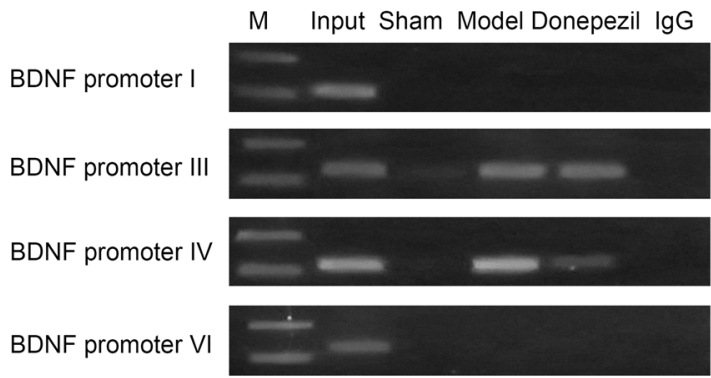

d

Hippocampus BDNF promoter III

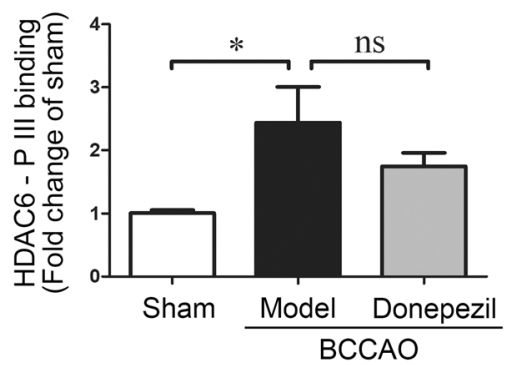

f

Hippocampus BDNF promoter IV

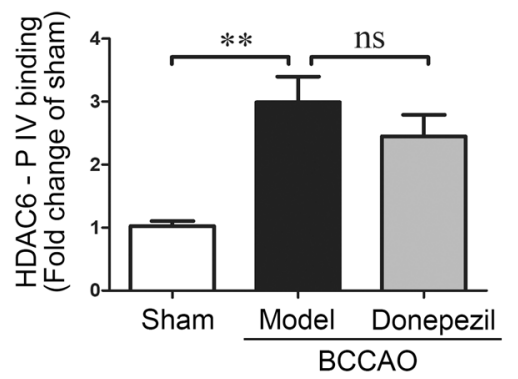

Fig. 4 The association of HDAC6 with BDNF promoter IV was regulated by donepezil. a, b Agarose gel images showing the association between HDAC6 and BDNF promoters P-I, P-III, P-IV, and P-VI. c-f qPCR analysis of BDNF promoter III and BDNF promoter IV binding to HDAC6 in the cortex and hippocampus. Significance was analyzed by one-way ANOVA. The data are shown as the mean \pm SEM. ${ }^{*} P<0.05$ and ${ }^{* *} P<$ 0.01 .

Donepezil regulated BDNF by reducing binding between HDAC6 and BDNF promoter IV

We previously indicated that donepezil increased BDNF expression in the cortex and hippocampus and that donepezil reduced the nuclear translocation of HDAC6 in the cortex without obvious changes in the hippocampus. To gain a deeper understanding of the regulation of BDNF by donepezil through HDAC6, we carried out ChIP to investigate the association between HDAC6 and BDNF promoters. As shown in Fig. 4a, b, HDAC6-immunoprecipitated and IgG-immunoprecipitated DNA were amplified by PCR against the BDNF promoters P-I, P-III, P-IV, and P-VI. HDAC6 showed no association with $\mathrm{P}-\mathrm{I}$ or $\mathrm{P}$-VI. P-III and P-IV were obviously associated with HDAC6 in the BCCAO model group in the cortex and hippocampus. P-III was associated with HDAC6 in the donepezil group in the cortex and hippocampus. P-IV was associated with HDAC6 in the donepezil group in the hippocampus, but no band was detected in the cortex. The results of PCR indicated that HDAC6 was associated with BDNF promoter III and BDNF promoter IV. We performed $\mathrm{QPCR}$ to further investigate the quantity of HDAC6 associated with BDNF promoter III and BDNF promoter IV. In the cortex, as reflected in Fig. 4c, e, the fold changes in BDNF promoter III and BDNF promoter IV were greater in the BCCAO model group than in the sham group (both
$P<0.01)$. Treatment with donepezil reduced the level of BDNF promoter IV compared with that in the BCCAO model group $(P<$ $0.05)$ but did not change BDNF promoter III $(P>0.05)$. In the hippocampus, as reflected in Fig. $4 d, f$, the levels of BDNF promoter III and BDNF promoter IV were enhanced in the BCCAO model group compared with the sham group (P-III: $P<0.05$, P-IV: $P<0.01)$. However, no significant difference in the associated of HDAC6 with BDNF promoter IV was observed in hippocampus upon treatment with donepezil $(P>0.05)$.

Donepezil reduced ROS and enhanced the phosphorylation of AMPK and AKT in the cerebrum

Previous results have shown no evidence that donepezil regulates BDNF via HDAC6 in the hippocampus. However, the mRNA and protein expression of BDNF in the hippocampus was increased in the donepezil group compared with the BCCAO model group, which implies that donepezil regulates BDNF through other pathways in the hippocampus. Oxidative stress is an important factor in neurodegeneration, and reactive oxygen species (ROS) are the main factors of oxidative stress. DHE can be oxidized by intracellular ROS and be incorporated into chromosomal DNA to produce red fluorescence [17]. The production of red fluorescence and the fluorescence intensity represent the levels of ROS. Figure. 5a shows 

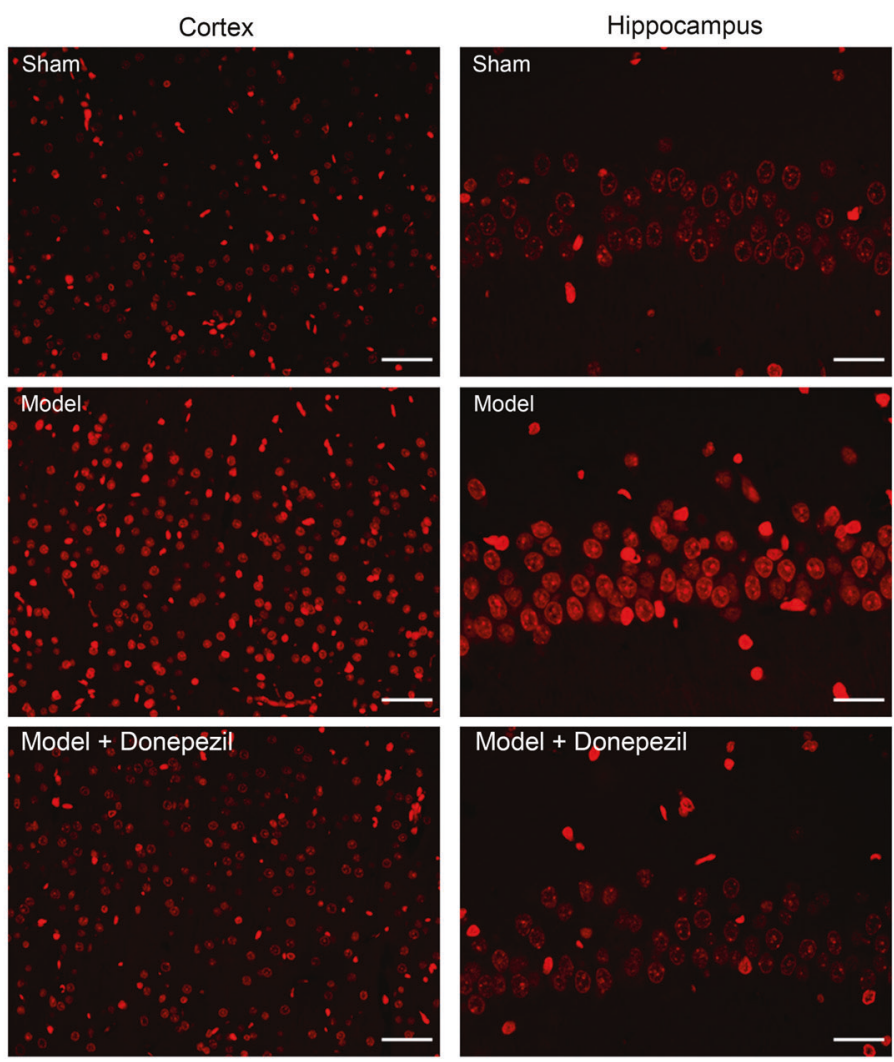

d

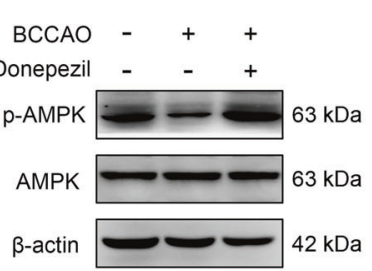

Cortex p-AMPK / AMPK

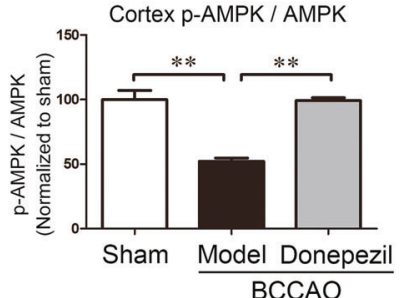

e

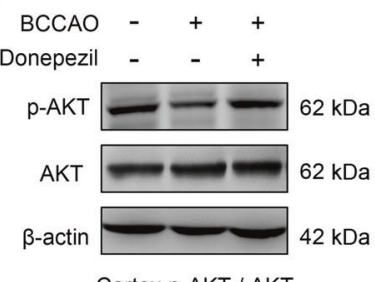

f

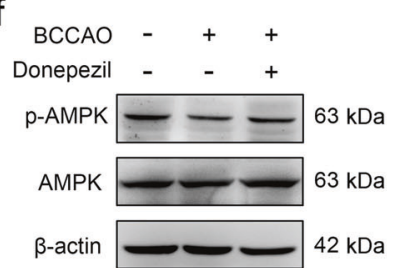

Cortex p-AKT / AKT

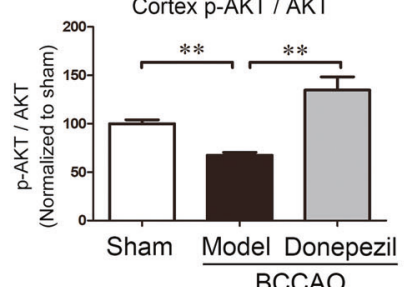

b

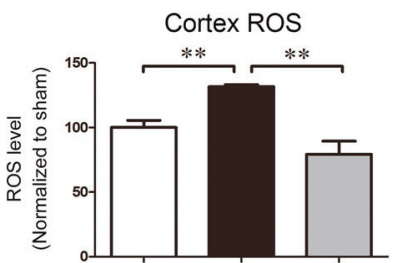

c

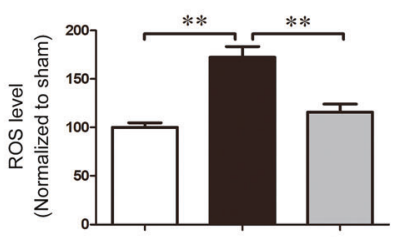

Fig. 5 Donepezil reduced ROS levels in BCCAO rats and activated AMPK and AKT in the cortex. a Representative images of DHE staining. All the images in the same region were captured on the same day under the same conditions with a fluorescence microscope. In the cortex, scale bar $=100 \mu \mathrm{m}$. In the hippocampus, scale bar $=50 \mu \mathrm{m}$. b, c ROS levels were measured based on fluorescence intensity and normalized to the mean of the sham group. $\mathbf{d}-\mathbf{g}$ The Western blotting results of the $\mathrm{p}$-AMPK/AMPK and $\mathrm{p}$-AKT/AKT ratios in the cortex and hippocampus. $\beta$-Actin was used as a reference protein, and protein expression was normalized to the mean of the sham group. Significance was analyzed by one-way ANOVA. The data are shown as the mean \pm SEM. ${ }^{* *} P<0.01$.

the representative fluorescence microphotographs of the cortex and hippocampus. In the cortex and hippocampus, obvious red fluorescence was detected in the model group, and donepezil treatment weakened the fluorescence. As reflected in Fig. 5b, c, compared with the sham group, the BCCAO group exhibited increased ROS levels in the cortex and hippocampal (both $P<0.01$ ). Treatment with donepezil reduced ROS levels compared with those in the BCCAO model group (both $P<0.01$ ).

AMPK and AKT are essential enzymes for neuronal activity, metabolism signaling pathways and the mediation of mitochondrial activity $[18,19]$. We examined $\mathrm{p}-\mathrm{AMPK}, \mathrm{AMPK}, \mathrm{p}-\mathrm{AKT}$, and
AKT protein expression in the cortex and hippocampus by Western blotting. As reflected in Fig. $5 \mathrm{~d}$, $\mathrm{f}$, in the cortex and hippocampus, BCCAO reduced the p-AMPK/AMPK ratio (both $P<0.01)$, and treatment with donepezil restored the level of phosphorylation of AMPK (both $P<0.01$ ). As shown in Fig. 5e, g, a reduction in the $\mathrm{p}$-AKT/AKT ratio was detected in the cortex and hippocampus in the model group (both $P<0.01$ ). However, treatment with donepezil only recovered the phosphorylation of AKT in the cortex $(P<0.01)$, and no change in the phosphorylation of AKT was detected in the hippocampus $(P>0.05)$. 


\section{DISCUSSION}

$\mathrm{CCH}$ has a high incidence in the elderly and is an important risk factor for AD and VD [2]. Donepezil is an FDA-approved drug for mild to severe Alzheimer's disease. Donepezil is a classic acetylcholinesterase inhibitor and can simultaneously increase the expression of BDNF in the CNS, thus enhancing neuronal activity and neuronal plasticity and protecting neuronal cells. Previous studies have indicated that donepezil can regulate the expression of BDNF by reducing miR-206-3p, a microRNA related to $B D N F$, thus protecting $A D$ transgenic mice [13]. However, in other studies, donepezil was demonstrated to play a neuroprotective role via ROS [20], the AMPK and AKT pathways [21] and other pathways. However, there has been no deeper investigation into the regulation of BDNF by donepezil. Based on recent studies, the expression of BDNF is regulated by the nuclear translocation of HDACs. In pathological situations, large amounts of HDACs are translocated to the nucleus. HDACs enter the nucleus and bind to BDNF promoter IV, which hinders the translation of BDNF and thus reduces the expression of BDNF in AD [14]. Therefore, we hypothesized that the mechanism of VD therapy could be explained by epigenetics. Our study aimed to investigate whether donepezil mediates BDNF via the regulation of the translocation of HDAC6 in VD.

BCCAO is a validated model for studying $\mathrm{CCH}$. According to studies of BCCAO, the acute phase (ischemia) occurs two to three days after the operation, while the chronic phase (oligemia) occurs eight weeks to three months after the operation. Previous studies have indicated that four weeks after BCCAO, rats in the BCCAO group, compared with rats in the sham group, show cognitive impairment in the MWM test $[22,23]$. In our study, donepezil was administered two weeks after the operation, and the treatment lasted for three weeks. Afer treatment, we evaluated cognitive impairment by the MWM test and step-down test. The experimental schedule simulated the early phase of vascular dementia and treatment with donepezil for mild cognitive impairment. The escape latency results showed that rats that underwent BCCAO surgery showed a statistical difference compared with the sham group from the 2nd day, indicating cognitive impairment in the BCCAO group. The donepezil-treated group showed a statistical difference compared with the BCCAO group from the 4th day, implying that donepezil restored long-term memory in $\mathrm{CCH}$. After 5 days of escape training, the donepezil group exhibited no statistical difference compared with the sham group in the number of platform crossings, which confirmed our belief that donepezil recovered long-term memory. Working memory was investigated by the step-down test. The results showed that the working memory of the BCCAO model was impaired and that donepezil improved working memory. The behaviors tests indicated the potential efficacy of donepezil in restoring longterm memory and attenuating working memory impairment.

The hippocampus is an important cerebral region responsible for learning and memory [24]. However, recent studies have indicated that the cortex also has functions in long-term memory [25-27]. Thus, our study focused on the effects of donepezil in the cortex and hippocampus in $\mathrm{CCH}$. According to results of Nissl staining, neurodegeneration was much more severe in the cortex than in the hippocampus. Treatment with donepezil attenuated neurodegeneration but failed to reverse neurodegeneration to the normal level. Golgi-Cox staining was performed to observe dendritic synapses. In our study, BCCAO decreased dendritic synapse density in the cortex and hippocampus, and donepezil restored the density to the level of that in the sham group. By comparing the results of Nissl staining and Golgi-Cox staining, we determined that donepezil was able to restore dendritic density to a normal level; however, it did not completely protect against neuronal degradation.

BDNF is a key factor in synaptic plasticity, and it can improve neural activity. In our study, BCCAO impaired the production of
BDNF, and donepezil restored the mRNA and protein expression of BDNF. The changes in BDNF were consistent with previous results of studies on neurodegeneration, so we believe that the regulation of BDNF by donepezil is a key factor in the attenuation of neurodegeneration in VD. Interestingly, the extent of restoration of BDNF expression in the cortex and hippocampus was not the same. The restoration of BDNF mRNA expression in that cortex was greater than that in the hippocampus (Supplemental Fig. 1C) $(P<0.01)$. Although no statistical difference in BDNF protein expression was detected between the cortex and hippocampus in the donepezil-treated group (Supplemental Fig. $1 d)(P>0.05)$, the mean level of BDNF protein expression was higher in the cortex. This suggested that the difference in the regulation of BDNF by donepezil in the cortex and hippocampus may occur at the transcriptional and translational levels.

Previous studies have proven that the nuclear translocation of HDAC6 can regulate the expression of BDNF [4, 14]. In our study, the distribution of HDAC6 was detected by immunofluorescence. BCCAO increased HDAC6 distribution in the nuclear area, while donepezil attenuated HDAC6 expression in the nucleus. By examining HDAC6 protein expression in the nucleus and cytoplasm, we found that BCCAO enhanced HDAC6 levels in the nucleus and that treatment with donepezil decreased the nuclear expression of HDAC6 in the cortex but had no significant effect in the hippocampus. We further extracted DNA precipitated with HDAC6 by ChIP. The results indicated that BCCAO increased the association of HDAC6 with BDNF promoter IV in the cortex and hippocampus. Donepezil attenuated the binding of HDAC6 to BDNF promoter IV in the cortex but had no significant effect in the hippocampus. Taken together, these results suggested that HDAC6 was translocated to the nucleus under the stress of $\mathrm{CCH}$ and that HDAC6 in the nucleus bound to BDNF promoter IV and eventually impeded the transcription of BDNF. Therefore, we believe that the differences in BDNF expression in the cortex and hippocampus were due to the different effects of donepezil on HDAC6 nuclear translocation.

In spite of the inability of donepezil to attenuate HDAC6 nuclear translocation, previous studies have showed that donepezil is able to enhance the expression of BDNF in the hippocampus, which convinced us that the regulation of BDNF by donepezil is not limited to HDAC6. In our study, the DHE staining results showed that donepezil attenuated ROS levels in the cortex and hippocampus. In addition, donepezil also reversed the suppression of p-AMPK induced by BCCAO in the cortex and hippocampus and the activation of $\mathrm{p}$-AKT in the cortex. However, donepezil failed to increase the p-AKT/AKT ratio in the hippocampus. Previous studies have also indicated that $\mathrm{p}$-AKT is decreased upon the knockdown of HDAC6 [28], which implies that AKT is associated with the regulation of HDAC6. However, further investigation is needed to confirm the relationship between HDAC6 nuclear translocation and p-AKT. The AMPK and AKT pathways are classic pathways involved in neural activity and energy metabolism. It has been proved that BDNF is also mediated by ROS, AMPK, and AKT [20, 21], and thus we believe that in $\mathrm{CCH}$, donepezil regulates BDNF not only by mediating HDAC6 nuclear translocation but also by reducing ROS levels and activating neuronal activity and energy metabolism. It is also worth noting that AMPK and AKT are not the only signaling pathways associated with ROS [29]. In our experiment, the expression of AMPK and AKT was detected to further confirm the formation of ROS. However, whether donepezil can affect ROS through other signaling pathways remains to be verified in future experiments (Fig. 6).

By comparing the expression of BDNF in the cortex and hippocampus, we found that the restoration of BDNF by donepezil was weaker in the hippocampus than that in the cortex. Taken together, the results suggested that donepezil was sensitive to ROS and enzymes associated with neural activity and energy 


\begin{tabular}{l}
\hline \multicolumn{1}{|c|}{ Vascular Dementia } \\
\hline - Neurodegeneration \\
- Increased HDAC6 nuclear translocation \\
- Reduced synapse dendritic density \\
- Induced ROS \\
- Suppressed AMPK \\
-Suppressed AKT \\
-Decreased BDNF
\end{tabular}

\begin{tabular}{l} 
Donepezil-treatment \\
\hline -Improved neuronal acitivity \\
-Decreased HDAC6 nuclear translocation \\
(in cortex) \\
- Restored synapse dendritic density \\
- Reduced ROS \\
-Activated AMPK \\
-Activated AKT (in cortex) \\
- Increased BDNF
\end{tabular}

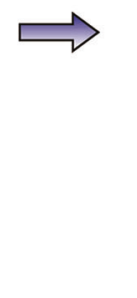

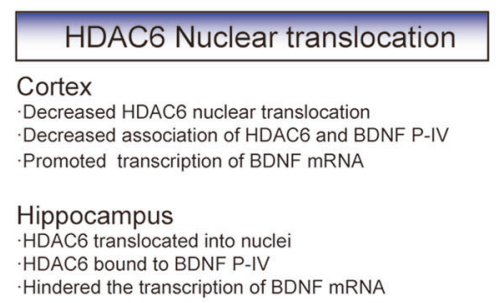

Hindered the transcription of BDNF mRNA

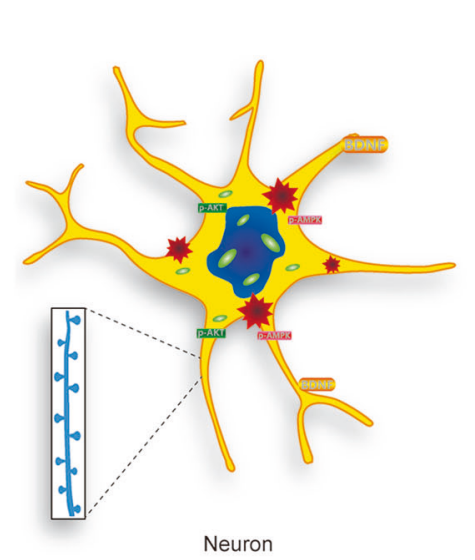

Neuron

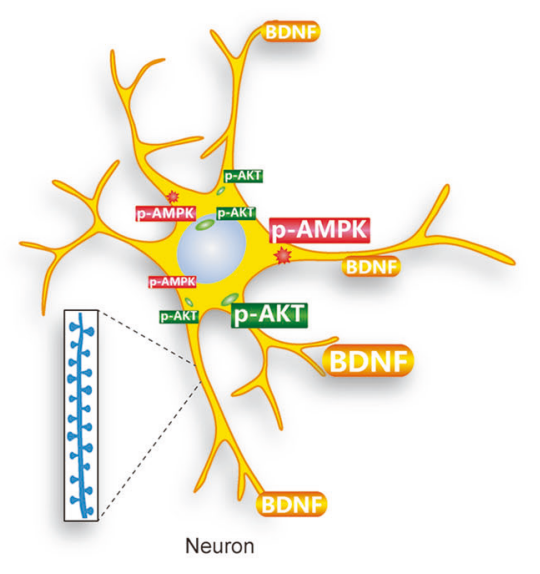

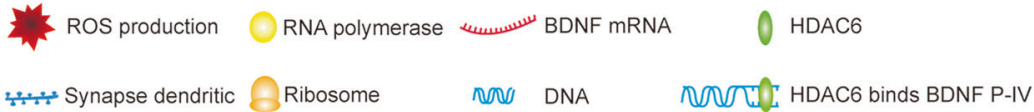

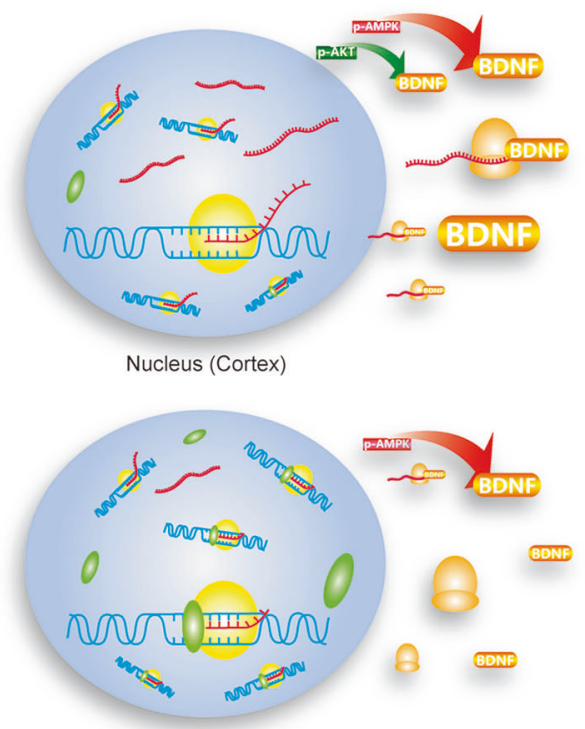

Nucleus (Hippocampus)

Fig. 6 Donepezil protected neurons and restored BDNF expression in the cortex and hippocampus. In vascular dementia, neurodegeneration, an increase in HDAC6 nuclear translocation, a reduction in dendritic synapse density, the suppression of AMPK and AKT, and a decrease in BDNF were detected in neurons. Treatment with donepezil in VD improved neuronal activity, decreased HDAC6 nuclear translocation in the cortex, restored dendritic synapse density, reduced ROS levels, activated AMPK, activated AKT in the cortex, and increased BDNF expression. HDAC6 nuclear translocation was different in the cortex and hippocampus. In the cortex, donepezil decreased HDAC6 nuclear translocation, decreased the association of HDAC6 with BDNF promoter IV, promoted the transcription of BDNF mRNA, and increased the expression of BDNF. Meanwhile, the activation of AMPK and AKT contributed to the increase in BDNF. In the hippocampus, donepezil did not affect the nuclear translocation of HDAC6 or the association of HDAC6 with BDNF promoter IV and hindered the transcription of BDNF mRNA. However, the activation of AMPK contributed to the expression of BDNF in the hippocampus.

metabolism, such as AMPK and AKT, in the cortex. Donepezil was given orally and entered the CNS through the blood. In BCCAO, the bilateral common carotid arteries are permanently ligated, but certain compensatory mechanisms, such as artery dilation, the recruitment of nonperfused capillaries and angiogenesis, maintain the blood supply to the cortex; however the blood supply to the hippocampus is weakened. The disparity of the blood supply to the cortex and hippocampus might explain why the cortex and hippocampus were affected differently by donepezil. Taken together, the results suggested that donepezil better reduced HDAC6 nuclear translocation in the cortex than in the hippocampus.

In our study, we discovered donepezil was able to attenuate neurodegeneration in VD. In addition, it reduced ROS levels and activated AMPK and AKT in the cerebrum. Interestingly, the effects of donepezil in the cortex and hippocampus were not fully consistent. In the cortex, donepezil reduced the nuclear translocation of HDAC6 and promoted the transcription of BDNF mRNA, eventually increasing the expression of BDNF; however, this process did not occur in the hippocampus, resulting in differences in BDNF recovery in the cortex and hippocampus. We hypothesize that in VD, the blood supply to different brain regions is different and that the efficacy of donepezil is dependent on blood supply to the cerebrum. For future drug development, increasing the distribution of donepezil in different brain regions and combining it with cerebrovasculoprotective drugs may improve the therapeutic effect of donepezil in VD. We believe that increasing BDNF via a reduction in the nuclear translocation of HDAC6 is a potential target for VD drug development.

\section{CONCLUSION}

In conclusion, donepezil was able to reverse cognitive impairment and memory dysfunction in VD. In the CNS, donepezil attenuated neuronal loss and degradation and protected dendritic spine synapses. Donepezil was able to reduce ROS levels and activated the AMPK and AKT pathways in the cerebrum. Interestingly, in our study, we discovered that donepezil enhanced the expression of BDNF by attenuating HDAC6 nuclear translocation in the cortex, but not in the hippocampus. In the hippocampus, donepezil was able to reduce ROS levels and activate AMPK, as in the cortex. The phosphorylation of AKT, which is regulated by HDAC6, was only activated in the cortex. Taken together, our results suggested that the effects of donepezil in the cortex and hippocampus in VD are different. Donepezil decreased HDAC6 nuclear translocation and increased BDNF expression in the cortex, but not in the hippocampus. This suggested that the regulation of BDNF via HDAC6 is a potential target for the treatment of VD. 


\section{ACKNOWLEDGEMENTS}

This work was supported by the National Natural Science Foundation of China (81603316, 81603315, 81503275, and 81560685), the CAMS Innovation Fund for Medical Sciences (CIFMS) (2016-I2M-1-004), and the Drug Innovation Major Project (2018ZX09711001-003-005, 2018ZX09711001-009-013).

\section{AUTHOR CONTRIBUTIONS}

WXJ and ZZ designed the study; WXJ, JHZ, and YP performed the research; $\mathrm{NHC}, \mathrm{ZZ}$, SFC, MZ, and QW contributed new reagents and analytical tools; WXJ and YP analyzed the data; WXJ and ZZ wrote the paper.

\section{ADDITIONAL INFORMATION}

The online version of this article (https://doi.org/10.1038/s41401-019-0334-5) contains supplementary material, which is available to authorized users.

Competing interests: All contributing authors have NO affiliations with or involvement in any organization or entity with any financial interest, or non-financial interest in the subject matter or materials discussed in this manuscript.

\section{REFERENCES}

1. Liu DD, Yuan X, Chu SF, Chen C, Ren Q, Luo P, et al. CZ-7, a new derivative of Claulansine $\mathrm{F}$, ameliorates $2 \mathrm{VO}$-induced vascular dementia in rats through a Nrf2mediated antioxidant responses. Acta Pharmacol Sin. 2019;40:425-40.

2. Singh $M$, Prakash A. Possible role of endothelin receptor against hyperhomocysteinemia and beta-amyloid induced $A D$ type of vascular dementia in rats. Brain Res Bull. 2017;133:31-41.

3. Barbay M, Taillia H, Nedelec-Ciceri C, Arnoux A, Puy L, Wiener E, et al. Vascular cognitive impairment: Advances and trends. Rev Neurol. 2017;173:473-80.

4. O'Brien JT, Thomas A. Vascular dementia. Lancet. 2015;386:1698-706.

5. Siuda J, Patalong-Ogiewa M, Zmuda W, Targosz-Gajniak M, Niewiadomska E, Matuszek I, et al. Cognitive impairment and BDNF serum levels. Neurol Neurochir Pol. 2017;51:24-32.

6. Aid T, Kazantseva A, Piirsoo M, Palm K, Timmusk T. Mouse and rat BDNF gene structure and expression revisited. J Neurosci Res. 2007;85:525-35.

7. Aarons T, Bradburn S, Robinson A, Payton A, Pendleton N, Murgatroyd C. Dysregulation of BDNF in prefrontal cortex in Alzheimer's disease. J Alzheimers Dis. 2019;69:1089-97.

8. Liu X, Zhang J, Sun D, Fan Y, Zhou H, Fu B. Effects of fluoxetine on brain-derived neurotrophic factor serum concentration and cognition in patients with vascular dementia. Clin Interv Aging. 2014;9:411-8.

9. Zheng $\mathrm{H}$, Niu S, Zhao H, Li S, Jiao J. Donepezil improves the cognitive impairment in a tree shrew model of Alzheimer's disease induced by amyloid-beta1-40 via activating the BDNF/TrkB signal pathway. Metab Brain Dis. 2018;33:1961-74.

10. Guida N, Valsecchi V, Laudati G, Serani A, Mascolo L, Molinaro P, et al. The miR206-JunD circuit mediates the neurotoxic effect of methylmercury in cortical neurons. Toxicol Sci. 2018;163:569-78.

11. Demyanenko S, Berezhnaya E, Neginskaya M, Rodkin S, Dzreyan V, Pitinova M. Capital ES, Cyrilliclass II histone deacetylases in the post-stroke recovery period- expression, cellular, and subcellular localization-promising targets for neuroprotection. J Cell Biochem. 2019;120:29266.

12. Graff J, Rei D, Guan JS, Wang WY, Seo J, Hennig KM, et al. An epigenetic blockade of cognitive functions in the neurodegenerating brain. Nature. 2012;483:222-6.

13. Choi H, Kim HJ, Kim J, Kim S, Yang J, Lee W, et al. Increased acetylation of Peroxiredoxin 1 by HDAC6 inhibition leads to recovery of Abeta-induced impaired axonal transport. Mol Neurodegener 2017;12:23. https://doi.org/10.1186/s13024017-0164-1.

14. Sen A, Nelson TJ, Alkon DL. ApoE4 and abeta oligomers reduce BDNF expression via HDAC nuclear translocation. J Neurosci. 2015;35:7538-51.

15. Tuvikene J, Pruunsild P, Orav E, Esvald EE, Timmusk T. AP-1 transcription factors mediate BDNF-positive feedback loop in cortical neurons. J Neurosci. 2016:36:1290-305.

16. Sangiovanni E, Brivio P, Dell'Agli M, Calabrese F. Botanicals as modulators of neuroplasticity: focus on BDNF. Neural Plast. 2017;2017:31-19.

17. Barzilai A, Yamamoto K. DNA damage responses to oxidative stress. DNA Repair. 2004;3:1109-15.

18. Zhao M, Li XW, Chen Z, Hao F, Tao SX, Yu HY, et al. Neuro-protective role of metformin in patients with acute stroke and type 2 diabetes mellitus via AMPK/ mammalian target of rapamycin (mTOR) signaling pathway and oxidative stress. Med Sci Monit. 2019;25:2186-94.

19. Liu S, Li F, Pan L, Yang Z, Shu Y, Lv W, et al. BRD4 inhibitor and HDAC inhibitor synergistically inhibit the proliferation of gallbladder cancer in vitro and in vivo. Cancer Sci. 2019;110:14102.

20. Rui M, Rossino G, Coniglio S, Monteleone S, Scuteri A, Malacrida A, et al. Identification of dual Sigma1 receptor modulators/acetylcholinesterase inhibitors with antioxidant and neurotrophic properties, as neuroprotective agents. Eur J Med Chem. 2018;158:353-70.

21. Atef MM, El-Sayed NM, Ahmed AAM, Mostafa YM. Donepezil improves neuropathy through activation of AMPK signalling pathway in streptozotocin-induced diabetic mice. Biochem Pharmacol. 2019;159:1-10.

22. Farkas E, Luiten PG, Bari F. Permanent, bilateral common carotid artery occlusion in the rat: a model for chronic cerebral hypoperfusion-related neurodegenerative diseases. Brain Res Rev. 2007;54:162-80.

23. Liu HX, Zhang JJ, Zheng P, Zhang Y. Altered expression of MAP-2, GAP-43, and synaptophysin in the hippocampus of rats with chronic cerebral hypoperfusion correlates with cognitive impairment. Brain Res Mol Brain Res. 2005;139: 169-77.

24. Preston AR, Eichenbaum H. Interplay of hippocampus and prefrontal cortex in memory. Curr Biol. 2013;23:R764-73.

25. TP Todd, and DJ Bucci. Retrosplenial Cortex and Long-Term Memory: Molecules to Behavior. Neural Plast. 2015:2015:414173.

26. Niekisch H, Steinhardt J, Berghauser J, Bertazzoni S, Kaschinski E, Kasper J, et al. Learning induces transient upregulation of brevican in the auditory cortex during consolidation of long-term memories. J Neurosci. 2019;39:2499-18.

27. Spets DS, Jeye BM, Slotnick SD. Different patterns of cortical activity in females and males during spatial long-term memory. Neuroimage. 2019;199:626-34.

28. Zhang SL, Zhu HY, Zhou BY, Chu Y, Huo JR, Tan YY, et al. Histone deacetylase 6 is overexpressed and promotes tumor growth of colon cancer through regulation of the MAPK/ERK signal pathway. Onco Targets Ther. 2019;12:2409-19.

29. Moloney JN, Cotter TG. ROS signalling in the biology of cancer. Semin Cell Dev Biol. 2018;80:50-64. 